\begin{tabular}{|c|c|}
\hline Title & Microfabrication of an anodic oxide film by anodizing laser-textured al uminium \\
\hline Author(s) & Jha, Himendra; Kikuchi, Tatsuy a; Sakairi, Masatoshi; Takahashi, Hideaki \\
\hline Citation & $\begin{array}{l}\text { Journal of Micromechanics and Microengineering, 17(10), 1949-1955 } \\
\text { https://doi.org/10.1088/0960-1317/17/10/004 }\end{array}$ \\
\hline Issue Date & $2007-10$ \\
\hline Doc URL & http:/hdl.handle.net/2115/30212 \\
\hline Rights & Copyright $\odot 2007$ IOP Publishing Ltd. \\
\hline Type & article (author version) \\
\hline File Information & JMM17.pdf \\
\hline
\end{tabular}

Instructions for use 


\title{
Microfabrication of anodic oxide film by anodizing of laser-texture aluminium
}

\author{
Himendra Jha*, Tatsuya Kikuchi, Masatoshi Sakairi, \\ and Hideaki Takahashi
}

Lab. of Interface Microstructure Analysis (LIMSA),

Division of Materials Science and Engineering,

Graduate School of Engineering, Hokkaido University,

Kita-13, Nishi-8, Kita-Ku, Sapporo 060-8628, Japan.

E-mail: himendra@eng.hokudai.ac.jp or

kiku@elechem1-mc.eng.hokudai.ac.jp

\begin{abstract}
A simple method for the fabrication of microstructures of aluminium anodic oxide film (anodic alumina) by anodizing laser-textured aluminium is demonstrated. In the process, the aluminium substrate was first textured by a low power laser beam, and then the textured aluminium was subjected for anodizing, to develop a continuous, thick porous layer on the textured surface. Microstructures with a few to several tens of micrometers depth were fabricated successfully on the anodic oxide film using various combinations of laser power density and laser scanning speed. Removing the film from the aluminium substrate enables the fabrication of various 2D and 3D microstructures from anodic alumina.
\end{abstract}

\section{Introduction}

Fabrication of structures of ceramics and various types of glasses, polymers etc, in microand nano-scale play an important role in many areas of modern technology. Such microsized structures have been used to fabricate various types of micro-devices for optical, chemical, biological, medical and other applications in the rapidly growing tinytechnologies field [1-3]. 
The porous anodic oxide film formed on aluminium, prepared by anodizing in oxalic acid solution, is similar to leuco-sapphire or glass ceramics, which are widely used in microelectronics. Porous anodic oxide film has sufficient mechanical strength and stability in extreme environments, which makes it suitable for the subsequent fabrication of hybrid integrated circuits [4]. Taking advantage of the properties of anodic oxide film formed by anodizing, various applications have been reported in the past few decades [5-9]. Recently, thick porous anodic oxide film has been recognized as a promising material for the fabrication of micro devices [10-12]. The anodic oxide film of aluminium is transparent and can resist very high temperatures, therefore it may be a good candidate for the fabrication of micro components for photonic as well as for high temperature processes. In these contexts, fabrication of microstructures of anodic oxide film of aluminium, with a simple, precise, and cost-effective method is of great interest in many areas of microtechnology.

Photolithographic techniques are well suited for production large numbers of identical parts, as they are very efficient for high throughput processes. However, there are many types of geometries and miniaturized components that are not compatible or cost-effective if processed with photolithographic tools. In those cases, other techniques such as direct-write processes offer unique advantages and capabilities for the fabrication of miniaturized components $[13,14]$. Among directwrite processes, laser tools have been successfully used for the fabrication of miniature components [15-18]. The growing number of companies developing laser micromachining techniques for an expanding number of applications is proof that laser micromachining is a well-established fabrication technique.

The present research aims to establish a technique for the fabrication of microstructures of anodic alumina by anodizing of laser textured aluminium surfaces, followed by substrate dissolution. The effects of parameters like laser power density and laser scanning speed on the shape and size of the resulting microstructures are investigated. Additionally, microstructures of the anodic oxide film of aluminium are fabricated to demonstrate the capabilities of the technique.

\section{Experimental Methods}




\subsection{Specimen and pre-treatment}

Highly pure (99.99 wt.\%) 350 $\mu$ m thick aluminium specimens were ultrasonically cleaned in ethyl alcohol for 10 minutes. The specimens were immersed in $\mathrm{CH}_{3} \mathrm{COOH} 78$ vol. $\%+\mathrm{HClO}_{4} 22 \mathrm{vol} . \%$ solution at $283 \mathrm{~K}$, for $5 \mathrm{~min}$ with a constant voltage of $28 \mathrm{~V}$ to get the electropolished surface on aluminum [19]. The various steps preformed for the fabrication of microstructures on anodic oxide film by anodizing laser-textured aluminium is summarized in Fig. 1 (a).

(a)

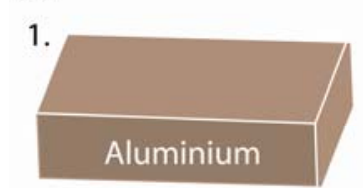

Electropolishing

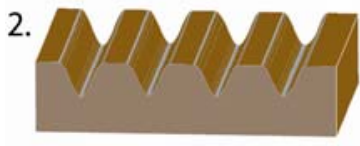

Laser-texturing

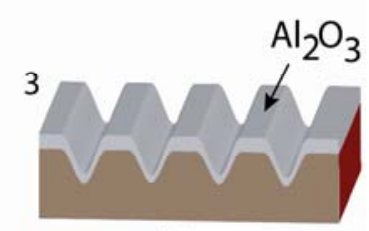

Anodizing

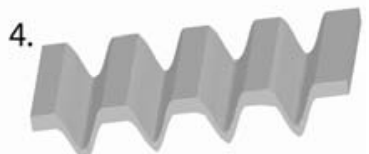

Al Removal

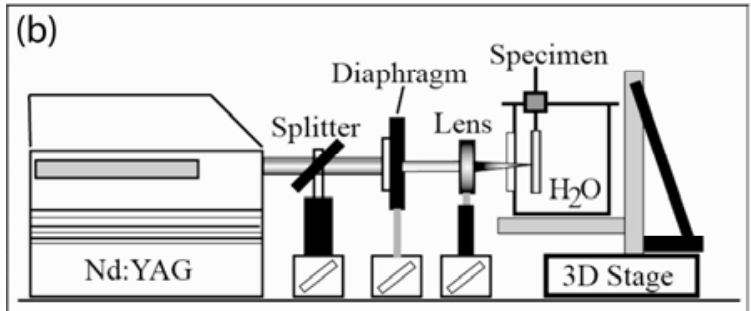

Figure 1: (a) Different steps for the fabrication of microstructures by anodizing of laser textured aluminium surface. (b) Laser irradiation setup for the texturing of the aluminium.

\subsection{Laser texturing}

The electropolished aluminium specimens were textured by a second harmonic, solid state, pulsed Nd:YAG laser (Spectra Physics; GCR-100 series) with $532 \mathrm{~nm}$ wavelength, $8 \mathrm{~ns}$ pulse width, and $50 \mathrm{~Hz}$ frequency. The laser set-up is shown in Fig. 1(b). The specimens were irradiated by laser inside a plastic cell filled with doubly distilled water, which placed on a movable stage (KOHZU; PK 569-B). Power density of the laser beam was measured by Ophir power measurement head (12A-P-SH), just before the laser irradiation. 


\subsection{Anodizing and aluminum removal}

The laser-textured specimens were anodized in $0.22 \mathrm{M} \mathrm{H}_{2} \mathrm{C}_{2} \mathrm{O}_{4}$ solution, at $293 \mathrm{~K}$ for 90 min (unless otherwise stated) with a constant current of $100 \mathrm{Am}^{-2}$. In some experiments, the specimens were subjected to pore-sealing in boiling doubly distilled water for $15 \mathrm{~min}$. The aluminium substrate was removed either by immersing in saturated $\mathrm{SnCl}_{4}$ solution or by amalgamating aluminium in saturated $\mathrm{HgCl}_{2}$ solution at room temperature. As the solutions does not affect the oxide film, a portion of aluminum should be exposed to solution (if completely bounded by the oxide film or others). Care should be taken while working with $\mathrm{HgCl}_{2}$ because of bad impacts on health as well as environment.

\subsection{Analysis}

The microstructures were analyzed by confocal scanning laser microscopy, CSLM (Lasertec, 1LM21D), and field emission scanning electron microscopy, FE-SEM (JEOL, JSM-6500F).

\section{Results and Discussion}

\subsection{Laser texturing on aluminium surface}

Microstructures of different dimensions and shapes were textured on aluminium by scanning a focused laser beam across the surface. The shape and size of the micro-textured structures can be controlled by varying the laser power density and laser scanning speed.

Figure 2 shows CSLM contrast images of the laser-textured microchannels on aluminium (a), and its three dimensional profile in (b). Three microchannels, 1, 2 and 3 were fabricated by using a $50 \mathrm{~mW} / \mathrm{cm}^{2}$ laser beam with scanning speeds of $20 \mu \mathrm{m} / \mathrm{s}, 30 \mu \mathrm{m} / \mathrm{s}$,

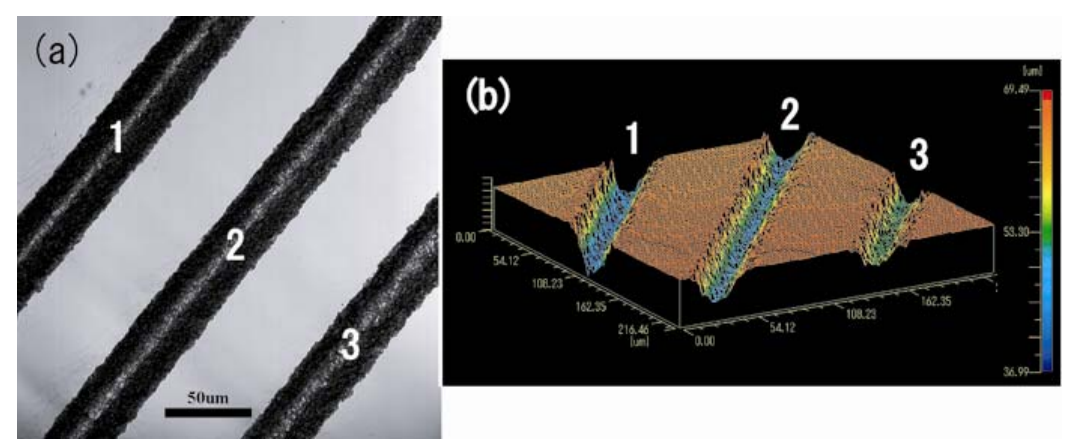

Figure 2: CSLM images of laser textured aluminium surface. (a) three microchannels 1, 2 and 3 fabricated with laser scanning speeds of 20, 30 and $40 \mu \mathrm{m} / \mathrm{s}$ respectively, at 50 $\mathrm{mW} / \mathrm{cm}^{2}$ of laser power density. (b) Three dimensional topographic image of the channels. 
and $40 \mu \mathrm{m} / \mathrm{s}$ respectively. It can be seen that, the microchannels have good edge definition and consistent shape throughout their length. Figure 2(b) shows that, increasing the scanning speed significantly decreases the depth and width of the texture, and there is a high degree of uniformity along a particular laser-textured microchannel. The variations in bottom-depth and surface-width of the textures with scanning speeds and laser power densities are plotted in Fig. 3. Fig 3(a) shows the depth at different scanning speeds, from 10 to $100 \mu \mathrm{m} / \mathrm{s}$, at two different laser power densities 50 and $100 \mathrm{~mW} / \mathrm{cm}^{2}$. Here, the depth decreases with increasing laser scanning speed, while it increases with laser power density. Increasing the power density from 50 to $100 \mathrm{~mW} / \mathrm{cm}^{2}$, the depth increases about two to three times. The surface-width, also, shows a similar trend, i.e. it decreases with increasing scanning speed and increases by increasing laser power density as indicated by Fig. 3(b).
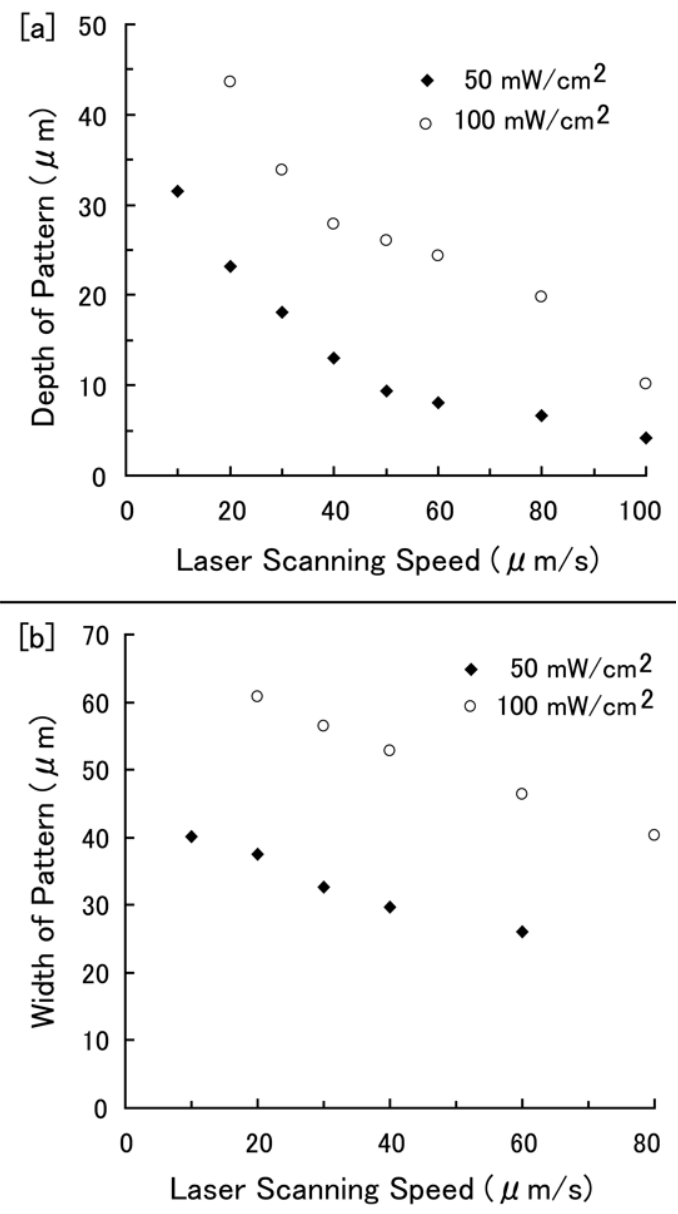

Figure 3: variation in (a) depth, and (b) width of laser-textured microchannels on the aluminium surface, with a laser scanning speed at 50 and $100 \mathrm{~mW} / \mathrm{cm}^{2}$.

It was found that, in the microstructures formed by relatively higher laser power densities (higher than $50 \mathrm{~mW} / \mathrm{cm}^{2}$ ) or with low laser scanning speeds, the edge of the 
microchannels are deformed by the edges extending outwards from the surface, as shown in Fig. 4 (a-c). Figure 4 (a) shows a microchannel formed by using a $50 \mathrm{~mW} / \mathrm{cm}^{2}$ laser beam with scanning speed $10 \mu \mathrm{m} / \mathrm{s}$. Here the edges of the channel are deformed evenly throughout its length. The cross-sectional height profile of the microchannel is shown in Fig 4 (b), which clearly shows the edges extending outwards from the top surface (by the deformed height "h"). Similarly, Fig. 4(c) shows a cross-sectional image of a microchannel formed by using a $100 \mathrm{~mW} / \mathrm{cm}^{2}$ laser beam at $30 \mu \mathrm{m} / \mathrm{s}$ of scanning speed. The height profile (b) and cross-section (c) both show that the shape of the microchannel is parabolic with an even ablated surface. It can be seen that the deformation takes place symmetrically at both edges of the channel.

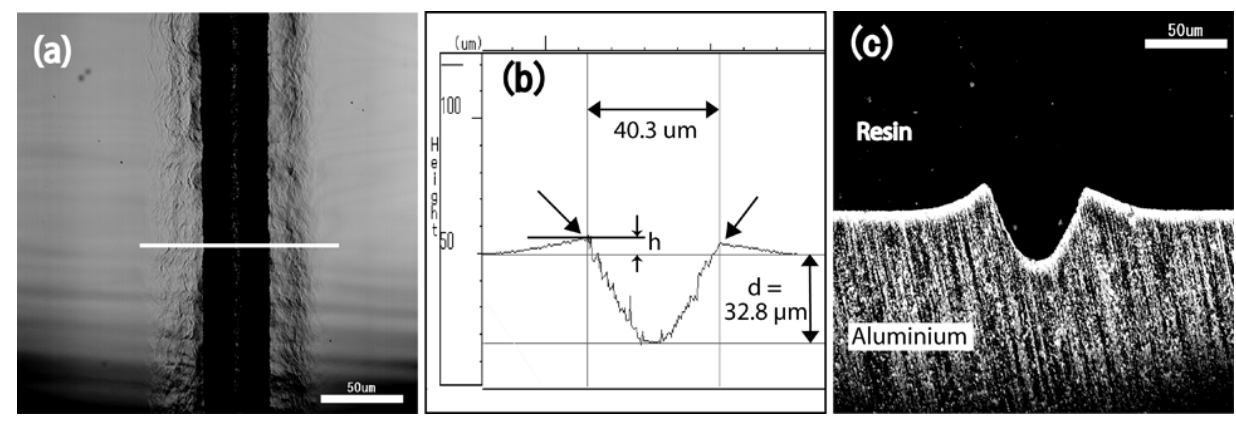

Figure 4: CSLM images of microchannel textured on aluminium surface. (a) Surface view of a microchannel textured by $50 \mathrm{~mW} / \mathrm{cm}^{2}$ laser beam with scanning speed $10 \mu \mathrm{m} / \mathrm{s}$; (b) Height profile of the microchannel, along the white line is shown in " a"; (c) cross-sectional image of a microchannel formed by $100 \mathrm{~mW} / \mathrm{cm}^{2}$ laser beam at $30 \mu \mathrm{m} / \mathrm{s}$ of scanning speed. Uniform ablated area as well as deformation at the edges can be clearly seen in Fig (b) and (c).

Scanning the laser beam across the aluminium surface forms a continuous groove on the irradiated surface by ablation. Controlling the movement and direction of the laser irradiation path, a variety of microstructures can be carved on the surface. Focusing even a very low laser power density $\left(50 \mathrm{~mW} / \mathrm{cm}^{2}\right)$, causes ablation of the aluminium surface. Microstructures of various dimensions can be fabricated by adjusting different combinations of laser scanning speed and laser power density, as shown in Fig.3. Either increasing the laser power density or slowing the scanning speed, the amount of energy deposited on the irradiated surface increases, hence a higher ablated volume is realized. The morphology of the microchannel strongly depends on the amount of energy deposited on the surface. A higher energy leads to the deformation of the microstructure due to outward displacement of the melted layer at the wall of the microchannel, forming a lip like structure along the channel (see Fig. 4). It is assumed that such a displacement of the melted layer is due to reacting-forces originating from the laser ablation process [20,21]. Therefore, the 
deformation is more pronounced with higher power densities and lower scanning speeds. It was found that the microstructures formed by higher scanning speeds ( i.e., $\geq 40 \mu \mathrm{m} / \mathrm{s}$ at $100 \mathrm{~mW} / \mathrm{cm}^{2}$, or $\geq 20 \mu \mathrm{m} / \mathrm{s}$ at $50 \mathrm{~mW} / \mathrm{cm}^{2}$ ) either had very low or no such edge deformation.

\subsection{Anodizing of laser-textured aluminium surface}

Anodizing of laser-textured aluminium substrate forms a continuous and thick anodic oxide film across the surface, resulting in a textured microstructure on the anodic oxide film of aluminium.

Figure 5 shows CSLM contrast images of cross-sections of laser-textured aluminium substrate after anodizing. Figure 5 (a) shows the cross-section of microchannels fabricated by texturing with a $50 \mathrm{~mW} / \mathrm{cm}^{2}$ laser beam on aluminium, at a scanning speed of $40 \mu \mathrm{m} / \mathrm{s}$, followed by $90 \mathrm{~min}$ of anodizing. Anodizing forms a continuous, thick anodic oxide film uniformly over the laser irradiated as well as the non-irradiated aluminium surface. Even on the deformed-edge of the microchannel, a continuous and thick

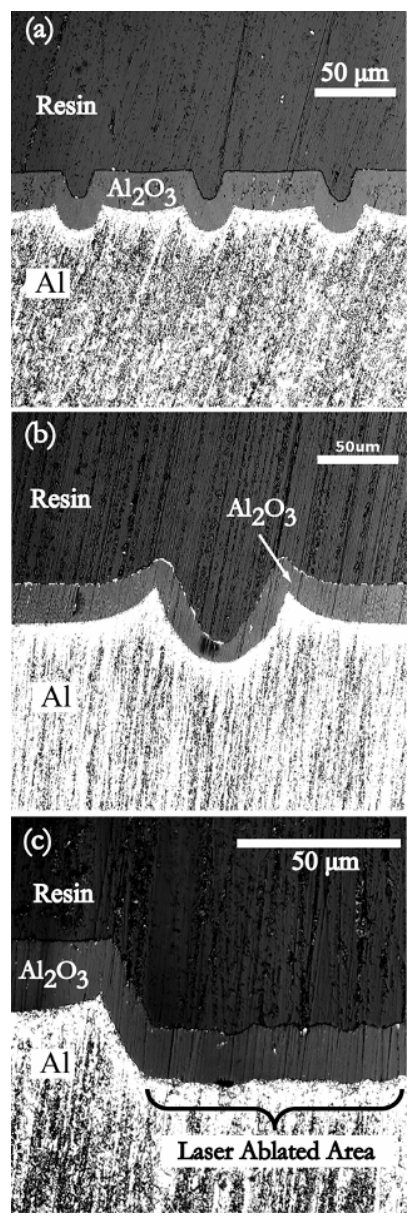

Figure 5: CSLM cross-sectional images of microstructures after 90 min anodizing of laser textured aluminium surfaces. (a) Anodic oxide film formed over laser textured microchannel, fabricated by using $50 \mathrm{~mW} / \mathrm{cm}^{2}$ laser beam with scanning speed $40 \mu \mathrm{m} / \mathrm{s}$. (b) Anodic oxide film formed on an edge-deformed microchannel, fabricated by using $100 \mathrm{~mW} / \mathrm{cm}^{2}$ laser beam with scanning speed of $30 \mu \mathrm{m} / \mathrm{s}$. (c) Anodic oxide film over wide laser ablated area, fabricated by overlapping the laser $\left(50 \mathrm{~mW} / \mathrm{cm}^{2}\right)$ irradiated part with scanning speed of $30 \mathrm{um} / \mathrm{s}$.

porous anodic oxide film is formed as shown in Fig. 5(b); Fig. 5(b) was fabricated after 90 min of anodizing of aluminium, textured with a $100 \mathrm{~mW} / \mathrm{cm}^{2}$ laser beam at $30 \mu \mathrm{m} / \mathrm{s}$ of 
scanning speed. Similarly, on a wider laser ablated area, the anodic oxide film forms uniformly after anodizing as shown in Fig.5(c), which is a cross-section of a part of a microchamber textured by partly overlapping microchannels. Figure 5 (c) shows that the surface of the anodic oxide film formed over the laser textured surface is somewhat rougher than that formed on the electropolished area. Figure 6 shows the change in depth (d) and deformation height (h) of the microchannels, before and after anodizing for $90 \mathrm{~min}$, with the laser scanning speeds. The depth of the microchannels before and after anodizing does not change significantly even after 90 min of anodizing as shown in Fig. 6 (a). In Fig. 6 (b), the edge deformation height $(\mathrm{h})$ is plotted against laser scanning speed before and after anodizing for $90 \mathrm{~min}$, at 50 and $100 \mathrm{~mW} / \mathrm{cm}^{2}$ of laser power density. Here, generally the deformation height (h) decreases almost linearly with increasing scanning speed, and decreases slightly by the anodizing. The strong influence of the power density on the edge deformation height can be understood from the results in Fig. 6(b).
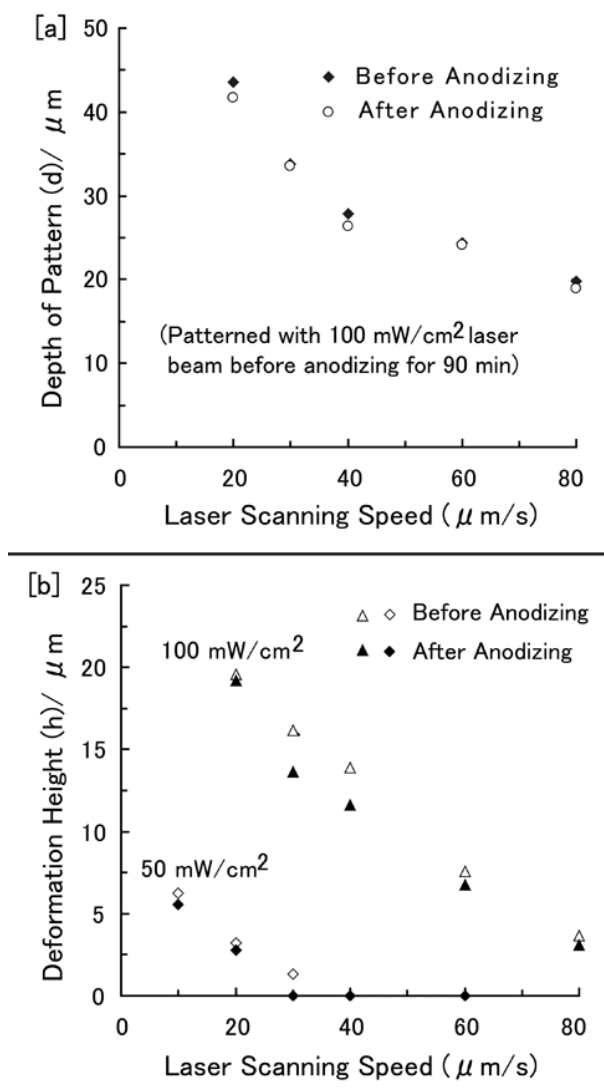

Figure 6: (a) Change in depth of laser-textured microchannels fabricated by $100 \mathrm{~mW} / \mathrm{cm}^{2}$ laser beam, before and after anodizing for $90 \mathrm{~min}$. (b) Variation in edge deformation height before and after anodization with scanning speeds at 50 and $100 \mathrm{~mW} / \mathrm{cm}^{2}$.

Figure 7 shows the FE-SEM images of microstructures on anodic oxide film of aluminium, formed after anodizing of laser-textured aluminium surfaces. Here, the 
microstructures were fabricated by texturing the aluminium surface by $50 \mathrm{~mW} / \mathrm{cm}^{2}$ laser beam and $30 \mu \mathrm{m} / \mathrm{s}$ scanning speed followed by $90 \mathrm{~min}$ of anodizing, before and after poresealing. Figure 7 (a) shows a microchamber formed after the anodizing of textured aluminium, without pore-sealing. The anodic oxide film uniformly and continuously formed over the whole surface (cross-section in Fig 5(c)). However, the surface morphology of the anodic oxide film at the laser treated area is somewhat different from that on the electropolished area. Different from the porous appearance, the oxide film surface at the laser irradiated part shows a very uneven appearance, as in Fig. 7 (b). Figure 7 (c) shows a similar microchamber, fabricated by anodizing followed by pore-sealing in boiling doubly distilled water for $15 \mathrm{~min}$. The magnified view of the film surface, formed on the lasertextured as well as on the non-textured aluminium, is shown in Fig. 7 (d). Here, there are foam-like (irregular patches) structures on the wall of the channel, whereas the non-textured area have uniform surface. These irregular patches covers all over the laser-textured area including the trench of the microchamber.
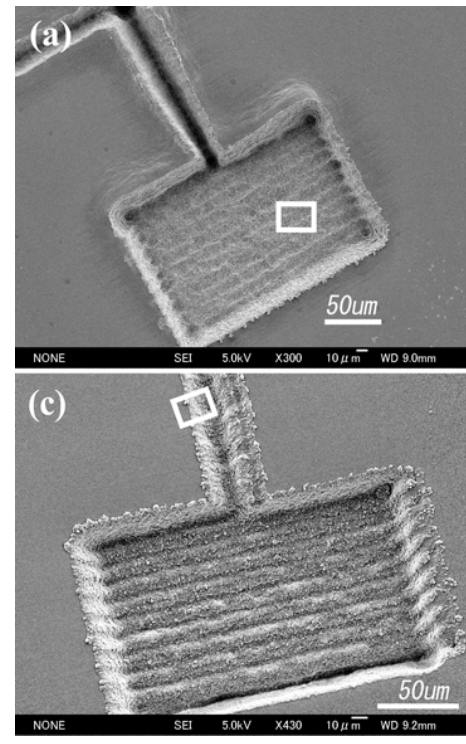

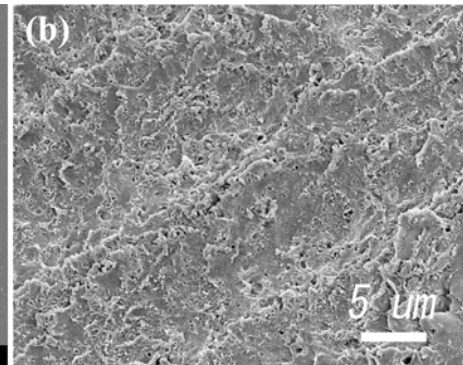

(d)

Figure 7: FE-SEM image of microchannel and microchamber fabricated by 90 min anodizing of the aluminium surface textured by $50 \mathrm{~mW} / \mathrm{cm}^{2}$ laser beam, with scanning speed of $30 \mu \mathrm{m} / \mathrm{s}$. (a) As formed microstructure after anodizing. (b) Magnified view of area indicated by rectangle in (a). (c) Microstructure after anodizing followed by pore sealing. (d) Magnified view of area indicated bv rectangle in (c).

Anodizing of aluminium in oxalic acid solution forms porous anodic oxide film over the surface. The thickness of the anodic oxide film can be controlled by adjusting the anodizing current or anodizing time. In our experimental setup $90 \mathrm{~min}$ of anodizing of a flat, electropolished aluminium surface gives an about $24 \mu \mathrm{m}$ thick, uniform anodic oxide film. 
Similarly, anodizing of the laser-textured aluminium, results in a continuous and uniform anodic oxide film on the laser ablated as well as on the bare surface. The formation of such an oxide film over the aluminium surface results in the texture observed on the film surface. The shape and size of the microstructure on anodic oxide film is almost the same as that fabricated on the aluminium surface. However, some deviation was observed in the case of very low laser scanning speeds and with a higher power beam. In these cases, the oxide film formed at the bottom of the microchannel was relatively thinner than on the surface. It is evident that, during laser texturing the focused laser beam interact with the surface of the aluminium, causing it to be heat-up, leading to the melting of the irradiated part. The melted part may be displaced or ejected from the irradiated part and solidify when it cools; in the same time debris formed all over the surface by solidifying the aluminium droplets and fragments. Such type of melting and re-solidification of the surface makes it uneven with different morphological structure than non-irradiated surface [22]. Anodizing of this uneven aluminium surface gives rise to the anodic oxide film with rough and irregular outer surface. Similarly, hydrothermal pore-sealing of porous anodic oxide film forms a highly crystalline hydroxide layer on the external surface of the film [8]. Since the oxide film surface on the laser-textured area is uneven, these parts develops irregular patches like hydroxide, as seen in Fig. 7 (d); whereas the non-textured surface (where the oxide film surface is smooth) have uniform hydroxide layer on the surface, as shown in the left part of the same figure. Though the pore sealing of the film enhances the strength of the microstructures, high surface roughness may limit its versatility in micro-fluidic applications.

Figure 8 shows FE-SEM images of microstructures on the anodic oxide film. The structures were textured by a $50 \mathrm{~mW} / \mathrm{cm}^{2}$ laser beam with $30 \mu \mathrm{m} / \mathrm{s}$ scanning speed, followed by anodizing for 90 min. Fig. 8 (a) shows a microstructure consisting of three microchambers connected to each other by microchannels. The depth of the channels is
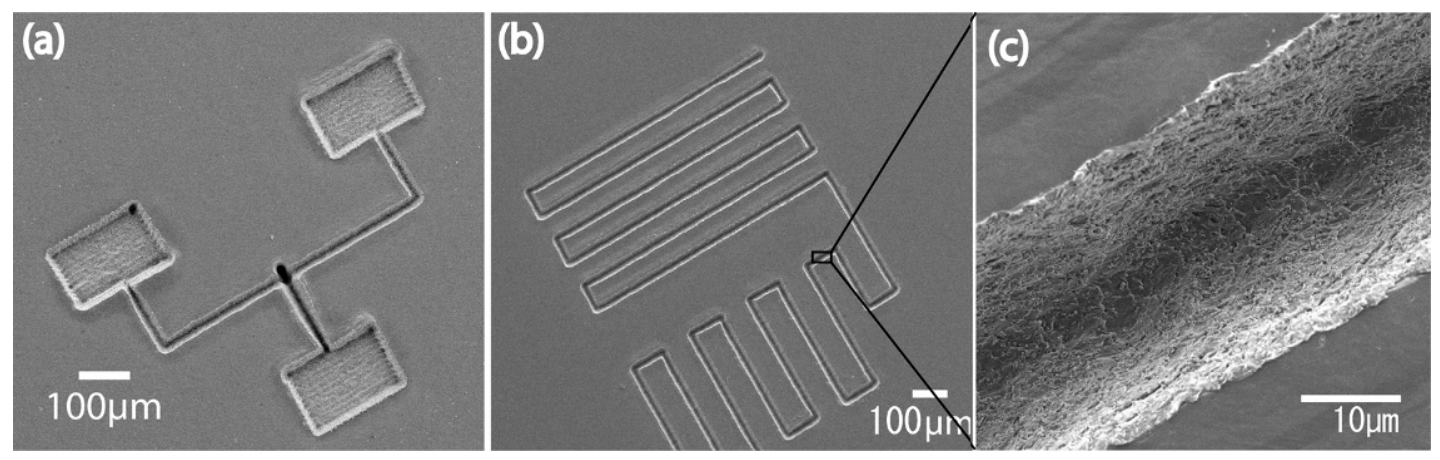

Figure 8: FE-SEM images of microstructures on anodic oxide film of aluminium fabricated by 90 min anodizing the aluminium surface textured by $50 \mathrm{~mW} / \mathrm{cm}^{2}$ laser

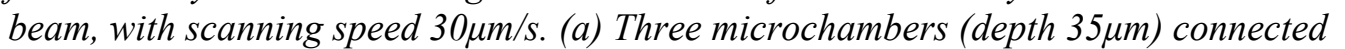
with microchannels (depth 27 $\mu \mathrm{m}$ ). (b) A long and continuous microchannel with depth $27 \mu m$. (c) Magnified view of a part of the microchannel in (b). 
$27 \mu \mathrm{m}$, while the depth of the chambers is about $35 \mu \mathrm{m}$. This is due to the partial overlap of the laser-irradiated area in the case of microchamber. Figure 8(b) shows a long and continuous microchannel of about $27 \mu \mathrm{m}$ depth. A magnified view of a portion of the microchannel is shown in Fig. 8 (c), showing that the channel has good edge definition and smooth inner surface. The depth at different parts of the microstructure can be changed by varying the scanning speed during the laser texturing on the aluminium surface. Repeating the laser scanning on already textured areas would further increase the depth at the site. Various types of microstructures can be fabricated on the anodic oxide film by texturing the aluminium substrate with laser beams followed by anodizing to the desired film thickness.

\subsection{Microstructure of anodic alumina}

Based on the textures on the aluminium surface, a wide variety of $2 \mathrm{D}$ and $3 \mathrm{D}$ microstructures of anodic oxide film (anodic alumina) can be obtained by anodizing of the textured-aluminium followed by removal of the remaining aluminium substrate.

Figure 9 (a) shows the FE-SEM image of closely-spaced parallel grooves on the anodic oxide film. The structure was formed by anodizing of a laser textured surface for 150 min followed by hydrothermal pore-sealing and then dissolution of the aluminium substrate. It can be seen that the microstructure is uniform without cracks or defects. The cross-section in Fig. 9(b) shows that the microstructure has a uniform shape with good edge definition. Similarly, micro-bumps on anodic oxide film were fabricated by anodizing of aluminium, consisting of laser-textured craters, as shown in Fig 10 (a). The micro-scale craters were first textured on electropolished aluminium surface, which on anodizing followed by aluminium dissolution forms micro-bumps on the back (toward the substrate), as shown in
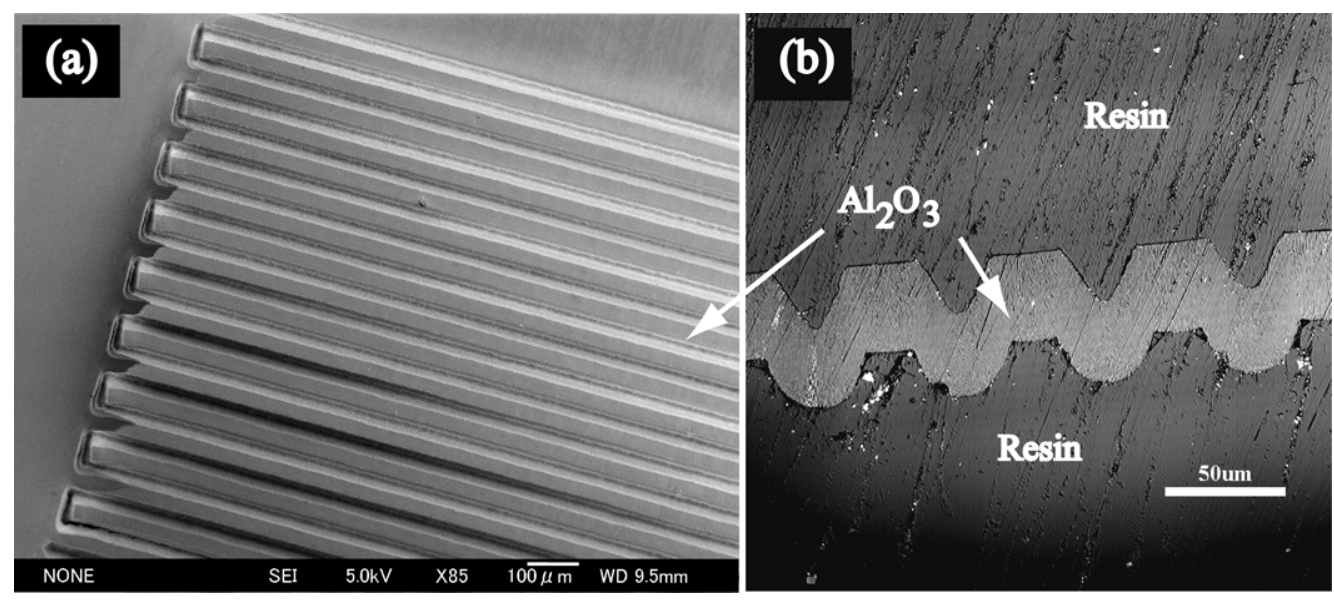

Figure 9: (a) Microstructure of anodic oxide film (anodic alumina) fabricated by anodizing and pore-sealing of laser textured aluminium surface followed by aluminium substrate dissolution. b) Magnified cross-sectional view of the microstructure in (a). 
Fig. 10(b). The diameter of the micro-bumps is about $40 \mu \mathrm{m}$ at about $200 \mu \mathrm{m}$ intervals. The magnified view of a micro-bump in Fig. 10 (c) shows a slightly irregular surface, due to the anodizing of the uneven laser irradiated surface. The isolated anodic oxide film is fragile in nature, and can easily break if not processed carefully. It was found that the thinner film (about less than $10 \mu \mathrm{m}$ ) was difficult to detach from the aluminium surface safely (unbroken), due to the fragile nature as well as relatively stronger anchoring at laser textured area than in smooth area.

The present method for the fabrication of microstructures of anodic oxide film of aluminium provides a simple and cost-effective approach to the fabrication of a variety of various $2 \mathrm{D}$ and $3 \mathrm{D}$ microstructures. The microstructures thus formed, after anodizing of the laser textured surface (Fig. 8) will be suitable for the fabrication of various micro-fluidic devices, here, the aluminium substrate attached to the anodic oxide film gives sufficient mechanical support for the devices. Moreover, removing the anodized film from the aluminium substrate provides a wide band-gap, transparent, and highly temperature resistant microstructures of anodic alumina. These types of microstructures would be useful in the design of various types of micro-components including photonic, high temperature packaging, high temperature micro-devices etc.

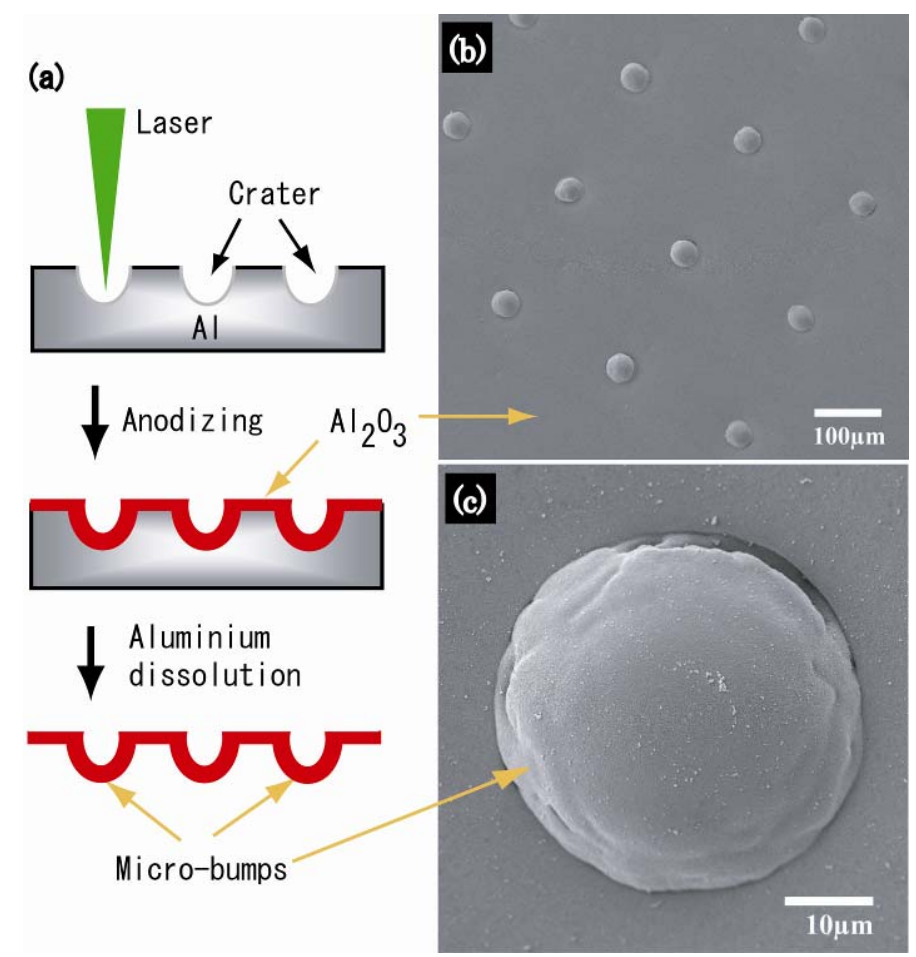

Figure 10: Fabrication of micro-bumps on the anodic oxide film of aluminium. (a) Schematic of the different steps; (b) micro-bumps on the anodic oxide film of aluminium, and (c) magnified view of a micro-bump. 


\section{Conclusions}

Microstructures can be textured on anodic oxide film of aluminium, by anodizing the laser-textured aluminium substrate. The shape and size of the microstructures significantly depends on the laser power density and laser scanning speeds. Increasing the power density or decreasing the scanning speed, the size of the microstructures increase, and deformation of the edges of the microstructures becomes significant in such cases. Microstructures, displaying uniformity over extended-lengths and a well ordered shape can be realized by this method. By removing the textured-anodized film from the aluminium substrate, various 2D and 3D transparent microstructures of anodic oxide film of aluminium can be fabricated with good precision.

\section{Acknowledgement}

The work is supported by a Grant-in-Aid (\#19206078) from the Ministry of Education, Culture, Sports, Science and Technology, Japan, and Light Metal Education Foundation of Japan.

\section{References}

[1] Spearing S M 2000 Acta mater. 48 179-196

[2] Liwe L A, Zhang W, An L, Shah S, Luo R, Liu Y, Cross T, Dunn M L, Bright V, Daily J W and Raj R 2001 American Ceramic Society Bulletin 8025

[3] Han M, Hyun D-H, Park H-H, Lee S S, Kim C-H and Kim C G 2007 J. Micromech. Microeng. 17 1184-91

[4] Mukhurov N I, Efremov G I and Kotova I F, 1997 Proc. of the Technical Digest of IVMC'97 (Korea) 617-21

[5] Jha H, Kikuchi T, Sakairi M and Takahashi H 2007 Electrochem. Commun. 71597

[6] Masuda H, Ohya M, Asoh H, Nakao M, Nohtomi M and Tamamura T 1999 Jpn. J. Appl. Phys. 38 L1403-05

[7] Martin C R 1994 Science 266 1961-66

[8] Jha H, Kikuchi T, Sakairi M and Takahashi H 2007 Electrochimica Acta 524724

[9] Kikuchi T, Akiyama Y, Ueda M, Sakairi M and Takahashi H 2007 Electrochimica Acta 524480

[10] Delendik K I and Voitik O L 2001 Proc. SPIE, MICRO/MEMS 4592-49

[11] Jha H, Kikuchi T, Sakairi M and Takahashi H 2007 Appl. Phys. A 88 617-622 
[12] Nadeem A, Mescher M, Rebello K, Weiss L W, Wu C, Feldman M and Reed M L 1998 Proc. of the $11^{\text {th }}$ Annual Int. Workshop on Micro Electro Mechanical Systems, MEMS 98 ( Germany) 274

[13] Pique A and Chrisey D B (eds) 2002 Direct-write technologies for rapid prototyping applications (Academic Press)

[14] Oh K H, Lee M K and Jeong S H 2006 J. Micromech. Microeng. 16 1958-66

[15] Osgood R M, and Gilgen H H 1985 Annual Review of Materials Science 15 549-76

[16] Hnatovsky C, Taylor R S, Simova E, Rajeev P P, Rayner D M, Bhardwaj V R and Corkum P B 2006 Appl. Phys. A 84, 47-61

[17] Arnold C B, Serra P and Pique A 2007 MRS Bulletin 3223

[18] Stuke M, Mueller K, Mueller T, Williams K, Oliver R, Ohlberg D A A, Fuhr G and Williams R S 2007 MRS Bulletin 3232

[19] Wernick S, Pinner R and Sheasby P G 1987 The Surface Treatment and Finishing of Aluminum and its Alloys (ASM International)

[20] Korner C, Mayerhofer R, Hartmann M and Bergmann H W 1996 Appl. Phys. A 63 123-31

[21] Fishburn J M, Withford M J, Coutts D W and Piper J A 2006 Appl. Surf. Sci. 252 5182-88.

[22] Dahotre Narendra B (ed.) 1998 Lasers in Surface Engineering (ASM International) 